UDC 811.131.1'367.622

DOI 10.18485/italbg.2016.1.4

Ignazio Mauro Mirto*

Università di Palermo

\title{
DELL'USO NON NUMERABILE DI NOMI NUMERABILI
}

\begin{abstract}
Nell'esaminare il tratto [ \pm numerabile] dei sostantivi, lo studio pone due questioni: (a) l'opposizione fra i valori del tratto è di natura sintattica o dipende dai referenti cui i nomi rimandano? (b) più in generale, può la realtà extralinguistica esercitare un'influenza diretta su quella linguistica? Nelle frasi analizzate, reperite in vari corpora, nomi tipicamente numerabili mostrano proprietà da non numerabili. Lo studio suggerisce la natura non assoluta dell'opposizione, che pare regolata da meccanismi sintattici piuttosto che da influenze dirette del mondo fisico su categorie linguistiche.

Parole chiave: nomi (non-)numerabili, nomi nudi, nomi predicativi, realtà (extra-) linguistica, referente.
\end{abstract}

\section{IMPOSTAZIONE}

Il seguente scambio fra Ada e Bice (nomi di fantasia), proveniente da un post del gruppo 'La lingua batte' di Facebook, svela una convinzione comune a moltissimi parlanti:

Ada: Mi hanno appena detto che, così come si dice vado a casa/scuola, si dice anche vado a studio (dal medico). Mi sembra un'assurdità, altrimenti dovremmo dire anche vado a università. Qualcuno mi illumina? Esiste una regola?

Bice: Casa/scuola sono edifici, lo studio è una parte dell'interno. Credo sia questa la differenza sull'uso delle preposizioni.

Le domande riguardano l'articolo che può ricorrere all'interno di certi sintagmi preposizionali. La sua assenza è percepita come impeccabile in espressioni come (vado) a casa, a scuola (rispetto a frasi quali vado alla casa, a una scuola). Di contro, essa viene avvertita come implausibile in

\footnotetext{
*ignaziomauro.mirto@unipa.it
} 
(vado) a studio (uno studio medico) ${ }^{1}$. La risposta di Bice ha un contenuto implicito. A suo avviso, la lingua italiana riflette una differenza esistente nel mondo fisico tra edifici, siti in spazi esterni, e costruzioni ad essi interne: la prima condizione sarebbe codificata con assenza dell'articolo, la seconda invece lo imporrebbe. Bice ritiene così che certe regolarità riscontrabili nel mondo esterno si proiettino come altrettante regolarità nella lingua. Più in generale, crede che la realtà extralinguistica, l'organizzazione del mondo fenomenico, possa esercitare un'influenza diretta sulla realtà linguistica, fino a determinarne alcune categorie.

Il modo di considerare il rapporto tra le due realtà menzionate condiziona facilmente lo sguardo sui fatti linguistici². Qui ci interessiamo della categoria dei sostantivi, contraddistinta al suo interno da diversi tratti, per es. animato/inanimato oppure concreto/astratto, spesso ritenuti assoluti. Un caso molto discusso, quello tra nomi numerabili e non numerabili (d'ora in avanti [ \pm numerabile]), è sovente basato in letteratura su criteri interamente o prevalentemente semantici. Ne dà testimonianza la seguente definizione ad opera di Giannini (1989: 511)3: "[...] si dicono nomi di massa quei nomi che denotano referenti al cui interno non si possono individuare ed enumerare i singoli componenti: per es., latte, sabbia, acqua, caffè, ecc. [...] Nei numerabili, di contro, il referente è sempre di ordine singolativo: cfr. $i$ libri, dueltrelalcuni libri, ecc."4.

Nei termini posti da Giannini, l'opposizione affonda le proprie radici nel cosiddetto 'referente'. Pur nelle diverse interpretazioni di questo delicato e problematico termine della metalingua, che finiscono col produrre un labi-

${ }^{1}$ Come chiariscono alcuni dei commenti al post, vado a studio è espressione utilizzata nella zona di Roma.

${ }^{2}$ Si legga a questo proposito quanto scrive Janner (2015: 336) in un interessante studio sui nomi di marca: "Mentre nella bibliografia [il significato dei marchionimi] è correlato a un referente esterno, in base al quale sono stabilite diverse categorie nelle quali suddividere i nomi commerciali, qui si afferma che il significato - o, saussurianamente, il valore - del marchionimo è dato dall'insieme delle proprietà combinatorie che emergono dall'analisi morfosintattica della proposizione" (corsivo di chi scrive).

${ }^{3}$ La definizione oppone i 'nomi di massa' ai 'numerabili'. Impostarla in questi termini è però fuorviante. Lo sostiene Joosten (2003: 216): “the term count-mass distinction [...] is misleading, since it incautiously takes together a primarily grammatical criterion (the (non-)countability of nouns) with a non-grammatical, ontological criterion (the denotation of mass vs. discrete entities)". La categoria 'nomi di massa', per di più, non dice nulla dei sostantivi astratti (v. sezione 2.1), questione che rimane irrisolta nella definizione proposta da Giannini.

${ }^{4} \mathrm{Su}$ sostantivi come sabbia, esempio diverso dagli altri perché concreto e con denotato in cui è in linea teorica possibile separare i singoli componenti, si veda quanto afferma Cruse (2000: 270). 
rintico gioco di specchi, tali definizioni creano un ponte, un collegamento, tra la realtà linguistica e quella extralinguistica ${ }^{5}$.

La complessa questione del rapporto tra le suddette realtà è da lungo tempo all'attenzione degli studiosi. Per ciò che riguarda il tratto [numerabile], Chierchia (2010: 101) l'ha recentemente posta nel modo seguente (la metalingua usata dall'autore rinvia ancora alla nota 3): "An old and difficult issue is whether the mass/count distinction is linguistic (stemming, that is, from grammar) or extralinguistic (i.e. rooted in language independent cognitive systems or, if you wish, in reality as such)."

Se Bice prende implicitamente posizione rispetto alla questione, l'opinione di Giannini (1989), pur se espressa in gergo tecnico, non ne pare distante, giacché il valore del tratto [numerabile] è detto essere in diretta dipendenza del referente cui il nome rimanda.

In queste pagine si esamineranno alcuni sostantivi che le nostre conoscenze del mondo inducono a classificare come numerabili e che nella stragrande maggioranza dei casi ricorrono effettivamente come tali. Tuttavia, il loro uso come non numerabili in frasi attestate, reperite in letteratura, sceneggiature, oppure in rete, mostra come, sia nel profano che nello studioso, le intuizioni basate sulla nostra esperienza sensibile, e ogni conclusione grazie ad esse raggiunta, creino, con le parole di Rosen (1991: 125; v. infra), una mera illusione. In linea con quanto affermato per l'inglese da Allan (1980: 541): "The customary disjunctive marking of lexical entries for English nouns as [ \pm countable] does not match the fact that the majority can be used both countably and uncountably in different NP environments", l'analisi conduce alla questione, di più ampio respiro, del rapporto tra la realtà extralinguistica e quella linguistica e intende mostrare per il tratto [numerabile] la problematicità delle analisi basate sul primo tipo di realtà fatte allo scopo di rendere conto delle caratteristiche del secondo.

\section{DISAMINE}

Si inizi il percorso con l'esame di alcuni sostantivi astratti, esemplificati perlopiù come numerabili nei dizionari da noi consultati. Seguirà l'analisi di sostantivi con riferimento comunemente concreto.

${ }^{5}$ Le seguenti considerazioni di Crystal (1987: 102) sembrano rilevanti: "Semantics is not directly concerned with the study of the external world. The world of non-linguistic experience is the province of physicists, geographers, psychologists, and others. Nor [...] is semantics easily able to cope with the study of how language refers to this external world - the notion of 'reference"' (corsivo nell'originale). 


\subsection{Astratti}

In italiano, un nome numerabile può ricorrere con l'articolo indeterminativo oppure flettere al plurale. Riguardo a queste proprietà, il sostantivo dubbio si comporta senz'altro da numerabile (Ho un dubbio, Ho numerosi $d u b b i)$. Un'ulteriore proprietà che distingue i numerabili dai non numerabili riguarda l'articolo partitivo. Questo si combina con il primo tipo di sostantivi obbligatoriamente al plurale (Mario desidera delle pinze vs. *Mario desidera della pinza), mentre il secondo ricorre al singolare (Mario desidera del latte). Anche sotto questo aspetto dubbio si comporta normalmente come numerabile (Mario ha dei dubbi). Tuttavia, la frase in (1), di Leonardo Sciascia $^{6}$, ne mostra un uso diverso ${ }^{7}$ :

(1) E credo che su questo punto anche Terranova avesse del dubbio

Come si vede, Sciascia adopera l'articolo partitivo lasciando dubbio al singolare, un fatto che allinea il sostantivo ai non numerabili, parallelamente a ciò che accade con i nomi di massa.

Nello stesso solco può collocarsi l'uso di pettegolezzo, un sostantivo più frequente al plurale che al singolare (Google, febbraio 2016), da parte di Ennio Flaiano in un suo noto aforisma:

(2) La pornografia è noiosa perché fa del pettegolezzo su un mistero

C'è poi il caso, diverso, di vita, anch'esso numerabile. Ne Il Gattopardo, però, Tomasi di Lampedusa si esprime come segue ${ }^{8}$ :

(3) Allora egli [Ciccio Tumeo] trascorreva vita felice perché il più fuggevole sorriso di un nobiluomo era sufficiente a riempire di sole una intera sua giornata

Mentre Leonardo Sciascia ne fa uso nella frase seguente9:

(4) Con mio padre spesso pensavamo di far vita qui

Diversamente dai casi con articolo partitivo prima esaminati, il sostantivo vita, in posizione post-verbale (e con ruolo molto probabilmente

${ }^{6}$ In L. Sciascia, Opere. 1971-1983, a cura di C. Ambroise, Bompiani, Milano, 1989, p. 750. Si noti che, a differenza di autentici nomi di massa come latte, in (1) l'articolo partitivo non può essere omesso con la stessa facilità (Hai latte vs. ??Credo che su questo punto anche Terranova avesse dubbio).

${ }^{7} \mathrm{Da}$ non trascurare altri casi, di diversa natura, in cui dubbio, se inserito in certi sintagmi preposizionali, può solo ricorrere al singolare, come per Sono in dubbio vs. *Sono in dubbi.

${ }^{8}$ Centesima edizione, 2014, Feltrinelli, Milano, pag. 125.

${ }^{9}$ In L. Sciascia, Opere. 1984-1989, a cura di C. Ambroise, Bompiani, Milano, 1989, p. 751 . 
predicativo), ricorre in entrambi i casi al singolare e ad articolo zero. Si noti, tuttavia, una differenza: in (3) l'inserimento dell'articolo indeterminativo lascerebbe la frase ben formata, mentre in (4) la guasterebbe irrimediabilmente. In nessuno dei casi, poi, può ricorrere l'articolo partitivo.

\subsection{Tipicamente concreti: casa $^{10}$}

L'uso non-numerabile dei sostantivi dubbio, pettegolezzo e vita si potrebbe attribuire al loro tratto [+ astratto]: in assenza di un referente concreto, un sostantivo godrebbe cioè di una certa flessibilità. Ora, immaginando vera l'influenza del mondo fisico su quello linguistico, la stessa flessibilità non dovrebbe riscontrarsi con sostantivi in grado di denotare entità concrete. È però facile mostrare che anche i nomi tipicamente concreti presentano duttilità riguardo al tratto in questione. Un test che consente di osservarla è quello della modificazione del nome per mezzo di un quantificatore (si tratta di una variante del test dell'articolo partitivo esemplificato sopra). Con il pronome interrogativo quanto, per esempio, un nome numerabile ricorre normalmente al plurale (cfr. (5)), mentre un nome di massa si dispone al singolare (cfr. (6)):

(5) a. Quante pinze vuoi?

b. ?Quanta pinza vuoi?

(6) a. Quanto zucchero vuoi?

b. ??Quanti zuccheri vuoi?

Con un sostantivo tipicamente concreto come casa ci si attenderebbe dunque un uso parallelo a quello in (5), un comportamento da nome numerabile. Ciò è senz'altro vero in molti casi, ma può capitare di imbattersi in ricorrenze come le seguenti (la frase (7) proviene dal doppiaggio italiano del film Killer élite, mentre (8) è tratta da Google):

(7) Devo ancora capire quanta casa c'è lì sotto

(8) Di quanta casa ha bisogno un uomo? (Anna Ruchat ${ }^{11}$ )

In combinazione con quanto, il sostantivo può ricorrere al singolare. Le frasi così mostrano che anche i nomi con riferimento generalmente concreto sono suscettibili di essere usati come non-numerabili. In altri termini, non si può stabilire alcuna relazione diretta tra l'opposizione concreto/astratto e

${ }^{10}$ Non si prenderanno in considerazione altri usi del sostantivo casa, già richiamati a proposito dello scambio in incipit, in cui il plurale non è consentito: Leo qui è di casa, Maria si trova a/in casa, Lo trovi per casa. Questo fenomeno, forse trascurato in letteratura, ha in italiano proporzioni vastissime (per uno di questi casi, v. Mirto (2013)).

${ }^{11}$ All'indirizzo: https://sconfinamento.files.wordpress.com/2011/12/inferno-snia. pdf (febbraio 2016). 
l'opposizione numerabile/non numerabile. Come è facile capire, tali usi non sono limitati al sostantivo casa (per casi simili in inglese, v. Allan (1980) e Cruse (2000: 270)) $)^{12}$.

\subsection{Tipicamente concreti: slittino}

Un altro sostantivo tipicamente concreto, slittino, reagisce prevedibilmente da numerabile in contesti quali quelli in (9):

(9) a. Desidera uno slittino (*Desidera slittino)

b. Possiede molti slittini

c. Vorrebbe degli slittini

Tuttavia, come si è già asserito in altra sede $\mathrm{e}^{13}$, in combinazione con fare (o praticare) il sostantivo può reagire in modo analogo a quello di un nome di massa. Può cioè entrare nella combinazione come [- numerabile], aprendo così la strada all'articolo zero. Tale uso comporta però un cambiamento semantico globale rispetto al valore in (9), giacché (10) vale 'Ugo pratica uno sport o un'attività fisica da fare con lo slittino':

(10) Ugo fa slittino

In presenza di un quantificatore, il sostantivo consente, a parità semantica con (10), il singolare, come illustra (11a), mentre la presenza dell'articolo indeterminativo produce un diverso costrutto, con effetti semantici globali (v. La Fauci 2011), come si può osservare in (11b), in cui il sintagma post-verbale è interpretabile come oggetto di creazione, dunque con fare fattitivo:

(11) a. Ugo fa molto slittino

b. Ugo fa uno slittino

\subsection{Tipicamente concreti: palo}

In aggiunta al più vasto significato di "legno avente sezione tondeggiante [...] piantato in terra" (dal Vocabolario Treccani, online), in italiano il sostantivo palo è usato anche per riferirsi al montante delle porte nei campi di calcio. Quest'ultima designazione sembra aver lasciato inalterato il valore del tratto [numerabile], visto che si trovano frasi come Nel secondo tempo l'attaccante ha fatto ben due pali, Quanti pali ha fatto? Ora, come si è già osservato, un sostantivo al singolare impiegato come numerabile impone normalmente la presenza di un determinante, come illustra la frase *Han-

${ }^{12}$ Come in (8), sembra plausibile, per esempio, una frase come Di quanto letto hai bisogno?, pronunciata da un coniuge il cui partner tendesse, durante il sonno, a invadere l'altrui spazio. Considerazioni analoghe valgono anche per l'esempio (5b).

${ }^{13}$ V. Mirto (2015). 
no piantato palo, mal costruita. Da verificare, quindi, il caso della celebre domanda in (12), tratta dal film Il secondo tragico Fantozzi:

(12) Chi ha fatto palo?

Il riferimento di palo in (12) potrebbe ancora essere interpretato come concreto (si pensi però a espressioni come fare argine, fare gavetta, fare ponte, fare salotto, fare strada). Ma il sostantivo, al singolare, si presenta ad articolo zero, dunque con una proprietà dei nomi con tratto [- numerabile] (e si noti la peculiarità di Chi ha fatto un palo?, che suggerisce scenari più complessi, con l'implicito che altri giocatori ne abbiano totalizzati o di meno o di più). Tale uso appare modellato sul caso di fare gol o fare rete, cioè di altri sostantivi caratteristicamente numerabili ma in grado di ricorrere al singolare (morfologicamente visibile solo in rete) come nomi nudi post-verbali. Rimanendo nel gergo calcistico, si osserva infine che in combinazione con altri verbi lo stesso fenomeno non può verificarsi: $L$ 'attaccante ha colpito il palo vs. *' ${ }^{\prime}$ attaccante ha colpito palo.

\section{CONCLUSIONI}

Si è osservato che un sostantivo solitamente numerabile, anche quando esso ha referente potenzialmente concreto, può alternativamente ricorrere con entrambi i valori del tratto [numerabile]. Lo dimostrano ulteriormente le frasi seguenti:

(13) a. Questa è una pietra

b. Questa è pietra

(13a) risulterebbe naturale al rinvenimento dell'oggetto che ha infranto il vetro di una finestra, con pietra che entra nella combinazione sintattica preceduto dall'articolo indeterminativo e dunque come numerabile. Quanto a (13b), la frase si potrebbe facilmente ascoltare nel caso di un carotaggio effettuato da geologi dopo aver ottenuto un campione dal sottosuolo. In (13b), dove ricorre ad articolo zero, pietra entra come non numerabile. Le frasi Quante pizze vuoi? e Quanta pizza vuoi? mostrano, grazie a un'operazione come quella già condotta in $(7)$ e (8), che la duplicità appartiene anche al sostantivo normalmente concreto pizza. Non tutti i sostantivi di questo tipo presentano però lo stesso grado di duttilità (si noti che, come in (13), anche in (14) il nome post-verbale è predicativo):

(14) a. Questa è una pistola

b. ??Questa è pistola

Si pensi, poi, al sostantivo cinema, che al singolare ha riferimento concreto in (15a), dove fare è fattitivo, ma non in (15b), assimilabile a un costrutto atto a esprimere una professione: 
(15) a. Quell'uomo farà un cinema

b. Quell'uomo farà cinema

Di fronte a duplicità come queste, e alle ricadute per niente trascurabili che esse hanno sul determinante del nome post-verbale, la definizione di Giannini in incipit mostra la corda.

I rapporti che intercorrono tra mondo linguistico ed extralinguistico sono da tanto tempo oggetto di analisi e su di essi sono stati versati fiumi di inchiostro. In un originale e suggestivo lavoro del 1991, Carol Rosen (1991: 125) definisce una illusion qualsiasi attribuzione di un valore assoluto, nella classificazione di un sostantivo tra i numerabili o i non numerabili, basata su conoscenze provenienti dall'esperienza sensibile ("world knowledge") ${ }^{14}$ : "[...] the fact that mud is a mass noun and shovel a count noun does correlate with my world knowledge. As a result, I experience the illusion that it is the outside world, not my L1 grammar, that makes popcorn a mass noun and peanuts a count noun" (sottolineatura di chi scrive).

Non si è inteso qui negare il fatto che un sostantivo con referente potenzialmente concreto possa ricorrere più da numerabile (opzione non marcata) che da non numerabile. È verosimile infatti che certe caratteristiche dei potenziali referenti (sempre in modelli del mondo reale, v. note 3 e 5) esercitino un'influenza sulla frequenza con cui un determinato sostantivo ricorre da numerabile. La tesi che si è difesa è un'altra: di nessun sostantivo numerabile si può escludere, a priori, un uso non numerabile, anche quando esso ha riferimento potenzialmente concreto. Un sostantivo come campeggio, per esempio, è frequentemente utilizzato come concreto. Quando ciò accade, è molto probabile che esso entri nella composizione sintattica come numerabile. Ma cosa dire di frasi come Marco fa campeggio ogni anno, in cui il sostantivo ricorre nudo (pur se l'articolo è consentito)? A noi sembra probabile un'analisi che veda campeggio come nome predicativo di una struttura con fare come verbo supporto. L'inserimento di un quantificatore consente ancora il singolare: Marco fa molto campeggio (cfr. sezione 2.1 ed esempio (11a)). A influenzare o determinare l'uso numerabile o meno, dunque, è cruciale il ruolo sintattico svolto dal sostantivo: se questo è predicativo, è possibile che esso entri nella composizione sintattica da non numerabile.

${ }^{14}$ Lo studio riguarda le difficoltà di apprendimento della lingua italiana da parte di discenti la cui lingua madre è l'inglese, in particolare per ciò che riguarda le valenze dei verbi detti psicologici. Secondo l'autrice, anche per le valenze di un predicato non ci si può affidare a conoscenze dipendenti dalla nostra esperienza sensibile (v. Mirto 2016). 


\section{BIBLIOGRAFIA}

Allan, K. (1980). Nouns and countability. Language, 56/3, 541-567.

Chierchia, G. (2010). Mass nouns, vagueness and semantic variation. Synthese, 174, 99-149.

Cruse, A. (2000). Meaning in Language. Oxford: Oxford University Press.

Crystal, D. (1987). The Cambridge Encyclopedia of Language. Cambridge: Cambridge University Press.

Giannini, S. (1989). Nome. In G. L. Beccaria (a cura di), Dizionario di linguistica e di filologia, metrica, retorica (pp. 510-513). Torino: Einaudi.

Janner, M. C. (2015). Sguardi linguistici sulla marca. Analisi morfosintattica dei nomi commerciali in italiano. Tesi di dottorato, Universität Zürich.

Joosten, F. (2003). Accounts of the count-mass distinction: A critical survey. Nordlyd, 31, 216-229.

La Fauci, N. (2011). Relazioni e differenze. Questioni di linguistica razionale. Sellerio: Palermo.

Mirto, I. M. (2013). Costrutti locativi e non-locativi con mettere. Romanistica Pragensia XIX, Les langues romanes à la lumière des corpus linguistiques, 71-90.

Mirto, I. M. (2015). Maniere di fare. Lessico e sintassi. Pisa: Edizioni ETS.

Mirto, I. M. (2016). Anatomia di un errore traduttivo. Valenze e predicati psicologici in inglese L1 - italiano L2. G\&D-Grammatica e didattica, 33-45. Disponibile al sito: http://www.maldura.unipd.it/GeD/DOCS/ Atti2014/Ignazio\%20Mauro\%20Mirto.pdf

Rosen, C. (1991). Relational Grammar: L2 learning and the components of L1 knowledge. In T. Huebner \& C. A. Ferguson (a cura di), Crosscurrents in Second Language Acquisition and Linguistic Theories (pp. 123-142). Amsterdam / Philadelphia: John Benjamins. 


\title{
UNCOUNTABLE OCCURRENCES OF COUNTABLE NOUNS
}

\begin{abstract}
Summary
In dealing with [ \pm countable] nouns, this study focuses on the following two questions: (a) does the binary feature depend on syntax or on the noun referents? (b) more generally, does extralinguistic reality exercise a direct influence over linguistic reality? The analysis addresses real sentences, drawn from literature, screenplays etc., in which typically countable nouns display properties of uncountable ones. We suggest that the selection of the features' value is governed by syntax rather than by real world characteristics able to effect linguistic categories.

Keywords: (un)countable nouns, bare nouns, predicate nouns, (extra)linguistic reality, referent
\end{abstract}

